\title{
Spreading and Solidification of a Molten Microdrop in the Solder Jet Bumping Process
}

\author{
Young-Soo Yang, Ho-Young Kim, and Jung-Hoon Chun
}

\begin{abstract}
This work develops a model to predict the spreading and solidification of solder droplets deposited on a solid pad in the solder jet bumping process. The variational principle is employed to solve the fluid flow and the semi-solid phase is modeled as a non-Newtonian slurry. This modeling greatly saves the computational expenses of conventional numerical procedures. The simulations reveal that the substrate temperature is the single dominant controlling parameter that determines the final bump diameter (or height) when the substrate possesses a high effusivity. When the effusivity of a substrate is relatively low, both the substrate temperature and the droplet temperature at impact play important roles in determining the final bump diameter. Our model can be used in designing the experimental conditions to find the optimal process conditions for a desired bump geometry.
\end{abstract}

Index Terms-Area-array packaging, recoiling, solder bump, variational principle.

\section{INTRODUCTION}

$\mathbf{T}$ HE interconnection technologies in the microelectronics industry have long been concerned with providing faster, smaller, and denser electronic components. To address these challenges, electronic packages have evolved from surface mount technology (SMT) and peripheral fine-pitch lead approach to area-array packaging (AAP) technology. AAP has many advantages over conventional techniques, in that AAP can provide higher I/O counts, greater density, lower cost, and higher performance as well as size reduction. For area-array packages, solder needs to be pre-deposited onto the packaging and this is commonly achieved through solder bumping [1]. Among solder bumping techniques, solder jet bumping, or solder jetting, is particularly promising because it lowers the manufacturing costs. However, its low throughput and irregular bump sizes have so far hampered its wide usage in practice. Therefore, accurate prediction and control of the solder bump shapes are crucial for the application of this technology to microelectronic components packaging.

In the solder jet bumping process, molten solder droplets ejected from a jetting device are deposited onto solid bonding pads of microelectronic circuits. The jetting device includes a crucible to contain molten solder and an orifice through

Manuscript received February 1, 2002; revised September 26, 2002. This work was recommended for publication by Associate Editor R. Lee upon evaluation of the reviewers' comments.

Y.-S. Yang is with the Department of Mechanical Engineering, Chonnam National University, Gwangju 500-757, Korea

H.-Y. Kim is with the Thermal/Flow Control Research Center, Korea Institute of Science and Technology, Seoul 136-791, Korea (e-mail: hoyoung@kist.re.kr).

J.-H. Chun is with the Department of Mechanical Engineering, Massachusetts Institute of Technology, Cambridge, MA 02139 USA.

Digital Object Identifier 10.1109/TCAPT.2002.806786 which solder droplets are ejected. The ejection is mostly driven by piezoelectric actuators nowadays (drop-on-demand mode). However, it is also possible to obtain a series of uniform droplets from a continuous jet by imposing periodic disturbance with a piezoelectric transducer (continuous jet mode) [2].

Collision dynamics and subsequent heat transfer associated with solidification determine the final shape of the solder droplets. Depending on the impact conditions, the molten droplet may exhibit various dynamic behavior until it completely solidifies. The heat transfer rate from the droplet to a target determines whether the droplet will rapidly freeze without recoil or simultaneously recoil and freeze. Rarely, when the target temperature is near the droplet's freezing point, high surface tension of the solder induces the droplet to bounce from the substrate.

Most modeling efforts to predict the solder bump shape have so far solved the full Navier-Stokes equation coupled with the energy balance [3]-[6], which procedure requires significant computational expenses. Hence a simpler model which can quickly evaluate the effects of varying process parameters on the solder bump shape is in great demand by industry. On the other hand, current approximate models which analytically predict the droplet shape, especially maximum spread diameter, are unable to adequately account for solidification effects and recoiling [6]-[9]. We note that recoiling is a characteristic feature in the deposition of molten metals with high surface tension and low viscosity.

Here we report a computational model for the spreading and solidification of solder droplets which greatly reduces the computational efforts to solve the Navier-Stokes and energy equations. Our fluid dynamic model is based on the variational principle. The method was proven to accurately predict the motions of liquid droplets upon impact with solid surfaces earlier [10]. To account for solidification during the droplet motion, we assume the droplet as a non-Newtonian slurry as observed in high temperature forming of metals [11]. These simplifying approaches are combined to evaluate the effects of various process parameters on final bump geometry (diameter and height). This is an extremely time-consuming and expensive task when rigorous numerical procedures are employed. Hence this work provides a useful tool for the design of solder bumping process by helping to find the optimal deposition conditions quickly.

\section{Modeling}

A fully molten droplet impacting upon a sub-cooled solid surface spreads and recoils until freezing halts its motion. As mentioned above, we model fluid flow and heat transfer during droplet spreading using simplified approaches rather than 
solving the full Navier-Stokes equation and energy equation. In this work, we use a fluid-dynamic model of Kim and Chun [10] based on the variational principle to simulate the droplet motion before the solidification sets in. Once the droplet temperature reaches the freezing point, a newly developed model simulates the motion of the semi-solid phase. We first present the fluid-dynamic model of Kim and Chun [10] briefly, and introduce the simplified heat transfer model to simulate the thermal states of the droplet and the substrate. Then we consider the droplet motion after its temperature reaches the freezing point by modeling the semi-solid phase spreading.

\section{A. Liquid Phase Spreading}

Kim and Chun [10] developed a model to simulate the droplet base diameter during its spreading and recoiling, based on the variational principle written as [12]

$$
\int_{t_{a}}^{t_{b}}\left(\delta T-\delta P+\delta W_{f}\right) d t=0
$$

where $T$ denotes the kinetic coenergy of the droplet, $P$ the surface potential energy, and $W_{f}$ the frictional work. It was necessary to assume the shape of the droplet during its motion, and both a cylinder and a truncated-sphere assumption were used in the modeling. It was shown that the Ohnesorge number defined as [10]

$$
O h=\frac{\mu}{\left(\rho \sigma D_{0}\right)^{1 / 2}}
$$

where $\mu$ is the viscosity, $\rho$ the density, $\sigma$ the surface tension, and $D_{0}$ the original diameter of the droplet, plays a dominant role in determining which shape assumption is more appropriate. The cylinder model was revealed to yield better predictions for liquids with a high surface tension and low viscosity, which requirements most liquid metals easily satisfy. Hence we use the cylinder model in this work. Fig. 1 shows the shape of a droplet assumed in the cylinder model. The simulation results of Waldvogel and Poulikakos [4], obtained by solving the full Navier-Stokes equation employing a finite element method, show that the solder droplet resembles a cylinder after being deposited on a solid surface. A detailed description for this modeling can be found in [10], thus here we present the results only briefly. When a cylindrical drop has a base diameter $D$ and the height $h$, the temporal evolution of $h$ is given by

$$
A(h) \ddot{h}-B(h) \dot{h}^{2}+C(h) \dot{h}+E(h)=0
$$

where the overdot denotes the time derivative. We give the dimensional forms in this paper while [10] uses nondimensional expressions. The coefficients are

$$
\begin{aligned}
A(h) & =\rho V\left(\frac{1}{3}+\frac{V}{8 \pi h^{3}}\right), \\
B(h) & =\frac{3 \rho V^{2}}{16 \pi h^{4}}, \\
C(h) & =\frac{F_{d} \mu V^{2}}{8 \pi \delta_{H} h^{4}}
\end{aligned}
$$

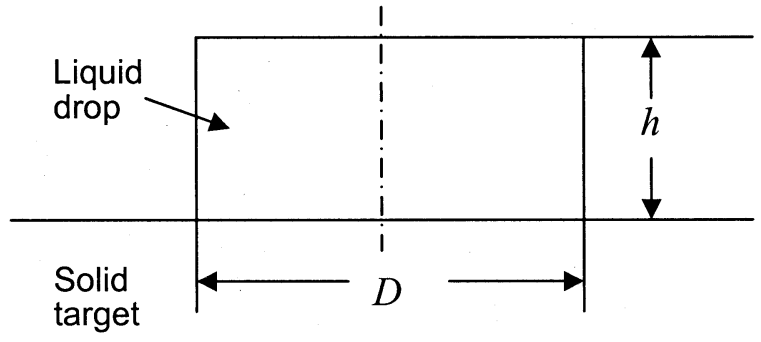

Fig. 1. Droplet shape assumed in the cylinder model.

and

$$
E(h)=\sigma\left[\left(\frac{\pi V}{h}\right)^{1 / 2}-(1-\cos \theta) \frac{V}{h^{2}}\right]
$$

where $V$ denotes the volume and $\theta$ the equilibrium contact angle of the droplet. The first two terms of (3) originate from the kinetic coenergy variation, and the third and the last terms correspond to the viscous work and the surface potential energy, respectively. We neglect the gravitational term in the potential energy since its relative magnitude to the surface energy, scaled as $(1 / 12) B o /\left(\eta_{\max }^{2}-1\right)$, is much less than one [10]. Here $B o$ denotes the Bond number defined as $B o=\rho g D_{0}^{2} / \sigma, g$ being the gravitational acceleration, and $\eta_{\max }$ is the ratio of the maximum spread diameter to the original droplet diameter.

The volume $V=\pi D^{2} h / 4$ remains constant throughout time. It is through this relation that either $D$ or $h$ completely describes the droplet geometry. The scale of the hydrodynamic boundary layer thickness, $\delta_{H}$, is written as

$$
\delta_{H}=\left(\frac{\mu^{2}}{\pi^{2} \rho \sigma D_{0}}\right)^{1 / 4} D_{0} .
$$

This corresponds to the characteristic thickness where the viscosity takes effect in an oscillating stagnation flow having the period $\left(\rho D_{0}^{2} / \sigma\right)^{1 / 2} \cdot F_{d}$ is the dissipation factor, which was taken to be 15 as in [10]. The initial conditions for $h$ and $\dot{h}$ at time $t=0$ are obtained by the following, respectively

$$
\frac{1}{4}(1-\cos \theta) D_{i}^{3}-D_{0}^{2} D_{i}+\frac{2}{3} D_{0}^{3}=0
$$

and

$$
\dot{h}(0)=-\left(\frac{U^{2}}{\frac{1}{3}+\frac{V}{8 \pi h^{3}(0)}}\right)^{1 / 2} .
$$

Here $D_{i}$ is the initial diameter of the cylinder, which is used to obtain the initial cylinder height by virtue of volume conservation. $U$ is the droplet velocity at impact. Equations (9) and (10) are derived by the equality of initial potential energy and kinetic coenergy, respectively, of the original droplet and those of a cylinder.

\section{B. Heat Transfer Model}

In the solder jetting process, a fully molten microdrop impacts on a solid bonding pad whose temperature is lower than the melting point of the droplet. Therefore, the conduction heat transfer from the droplet to the substrate should be considered to account for the microdrop solidification. Here we consider 
pure tin droplets which solidify at a single temperature. This approach can also be applied to other pure and eutectic materials. In this work, we construct a simple heat transfer model that is solvable without resorting to a complicated numerical procedure in line with the spirit using the variational principle for fluid flow modeling. In addition, we decouple the fluid flow and heat transfer problems so that whether the droplet is fully liquid or semi-solid, there is one differential equation to be solved for each thermal regime.

We assume a uniform temperature in the droplet, which is justified when the thermal gradient in the substrate is much greater than that in the droplet. This is easily satisfied when the droplet's superheat is moderate or when the substrate is relatively cold. It is because the ratio of the temperature gradient in the droplet to that in the substrate is approximately $\zeta=\left(T_{i}-T_{f}\right) /\left(T_{f}-T_{\infty}\right)$, where $T_{i}, T_{f}$, and $T_{\infty}$ are the initial droplet temperature, the freezing temperature, and the initial substrate temperature, respectively, that is $\zeta \ll 1$. Here we scaled the temperature difference in the drop liquid phase as $\left(T_{i}-T_{f}\right)$ since the temperature at the liquid/solid interface is kept constant at $T_{f}$. Moreover, the solder drop's Stefan number, $S t=c\left(T_{i}-T_{f}\right) / L, c$ and $L$ being, respectively, the specific heat and the heat of fusion, is indeed much smaller than the unity for superheat of less than $100{ }^{\circ} \mathrm{C}$. This implies that the release of latent heat, rather than sensible heat conduction, plays a major role in the drop solidification process.

Our modeling of the fluid flow is divided into two regimes, i.e., when the droplet is fully liquid and during solidification. A different formula for fluid flow should be employed when the droplet is partially solidified than for a fully liquid drop. Hence the time taken for a droplet to reach the freezing temperature should be estimated. When a droplet impacts on a sub-cooled solid target that is assumed as a semi-infinite body, the interface temperature is almost instantaneously raised to the freezing point of the droplet, $T_{f}$. The conductive heat transfer rate, $Q_{c}$, from the molten droplet to the substrate is given by [13]

$$
Q_{c}=k_{s} A \frac{T_{f}-T_{\infty}}{\left(\pi \alpha_{s} t\right)^{1 / 2}}
$$

where $k_{s}$ and $\alpha_{s}$ denote the thermal conductivity and the thermal diffusivity of the substrate, respectively, and $A$ the contact area of the droplet with the substrate. The time $t_{1}$, which designates the period the droplet cools to the freezing point from a super-heated state, is thus obtained by solving

$$
\int_{0}^{t_{1}} Q_{c} d t=\rho V c\left(T_{i}-T_{f}\right)
$$

where $c$ is the heat capacity of the droplet. To solve (12) combined with (11), we need to evaluate the contact area, or the cylinder base area in our model, with respect to time. The fluid flow and heat transfer analyses are decoupled to obtain a simple model, thus we temporarily assume that the cylinder diameter evolves as

$$
D=\frac{D_{i}-D_{m}}{t_{m}^{2}}\left(t-t_{m}\right)^{2}+D_{m}
$$

where $D_{m}$ is the maximum cylinder base diameter at $t=t_{m}$. This is a fairly close assumption for most droplet impact conditions with low $O h$ [10]. Substituting (13) into (11) and solving (12), we obtain the following algebraic equation for $t_{1}$ :

$$
\sum_{n=1}^{5} \beta_{n} t_{1}^{(2 n-1) / 2}=\frac{4 \rho V c\left(T_{i}-T_{f}\right)}{\pi^{1 / 2} \eta_{s}\left(T_{f}-T_{\infty}\right)}
$$

where $\eta_{s}$ denotes the effusivity of the substrate, $\eta_{s}=k_{s} / \alpha_{s}^{1 / 2}$. The coefficients are given by

$$
\begin{aligned}
& \beta_{1}=2 D_{i}^{2}, \\
& \beta_{2}=-\frac{8}{3} \frac{\left(D_{i}^{2}-D_{i} D_{m}\right)}{t_{m}}, \\
& \beta_{3}=\frac{1}{5} \frac{\left(12 D_{i}^{2}-20 D_{i} D_{m}+8 D_{m}^{2}\right)}{t_{m}^{2}} \\
& \beta_{4}=-\frac{8}{7} \frac{\left(D_{i}-D_{m}\right)^{2}}{t_{m}^{3}}
\end{aligned}
$$

and

$$
\beta_{5}=\frac{2}{9} \frac{\left(D_{i}-D_{m}\right)^{2}}{t_{m}^{4}}
$$

Next, we evaluate the semi-solid phase spreading time, $t_{2}$, designating a period during which the droplet's solid fraction increases from zero to one. The time is estimated in a similar manner to $t_{1}$, but in this case the heat loss of the droplet to the substrate is associated with the release of latent heat of the droplet

$$
\int_{t_{1}}^{t_{1}+t_{2}} Q_{c} d t=\rho V L
$$

Again assuming that $D$ follows (13), $t_{2}$ is obtained by the following algebraic equation:

$$
\sum_{n=1}^{5} \beta_{n}\left[\left(t_{1}+t_{2}\right)^{(2 n-1) / 2}-t_{1}^{(2 n-1) / 2}\right]=\frac{4 \rho V L}{\pi^{1 / 2} \eta_{s}\left(T_{f}-T_{\infty}\right)} .
$$

It is the effusivity, rather than the thermal conductivity, of the substrate that determines the solidification rate as shown in (14) and (16). It is emphasized that the assumption of (13) is used only to estimate $t_{1}$ and $t_{2}$, and the actual diameter is calculated based on the formula in the previous section (fluid flow analysis).

\section{Semi-Solid Phase Spreading}

When the droplet temperature reaches its freezing point, the droplet becomes semi-solid, and the fluid flow formulation as shown in (3) cannot be applied. At solid fractions between 0.05 and 0.1 , the slurry behaves as a non-Newtonian, history-dependent fluid [14]. At higher solid fractions above 0.6 , the slurry resembles a nonlinear viscoplastic solid as observed in high temperature forming of metals. In this work, we consider that the spreading stops at the solid fraction of 0.625 according to the results of [15]. In the variational formula we employ, the kinetic and surface potential terms are not affected by the existence of solid phase in the droplet. However, the dissipation term should 
be modified since the flow is no longer that of a Newtonian viscous fluid. The rate of energy dissipation, $\dot{e}$, for the semi-solid flow is expressed as [15], [16]

$$
\dot{e}=\frac{9}{16} \mu \dot{\gamma} \frac{\left(\frac{f}{f_{\max }}\right)^{1 / 3}}{1-\left(\frac{f}{f_{\max }}\right)^{1 / 3}}
$$

where $\dot{\gamma}$ is the shear strain rate, $f$ the solid volume fraction, and $f_{\max }$ the maximum solid volume fraction, 0.625 . We take the shear strain rate and the solid volume fraction as, respectively

$$
\dot{\gamma}=\frac{u_{r}}{h}
$$

and

$$
f=\frac{t-t_{1}}{t_{2}}\left(t_{1} \leq t \leq t_{1}+t_{2}\right)
$$

where the radial velocity $u_{r}$ is given by $u_{r}=-r \dot{h} / 2 h$ [10]. Substituting (18) and (19) into (17), we obtain

$$
\dot{e}=\frac{9}{64} \mu r^{2} \frac{\dot{h}^{2}}{h^{4}} a(t)
$$

where $a(t)$ is given by

$$
a(t)=\frac{\left(\frac{t-t_{1}}{0.625 t_{2}}\right)^{1 / 3}}{1-\left(\frac{t-t_{1}}{0.625 t_{2}}\right)^{1 / 3}} .
$$

Then the frictional work term, $\delta W_{f}$, is given by

$$
\delta W_{f}=\frac{9 \mu V^{2}}{128 \pi} \frac{\dot{h}}{h^{5}} a(t) \delta h .
$$

In a similar manner to that used in [10], we obtain the second order nonlinear differential equation based on the variational principle for $h$

$$
A(h) \ddot{h}-B(h) \dot{h}^{2}+\tilde{C}(h, t) \dot{h}+D(h)=0
$$

where all the coefficients remain the same as those in (3) except

$$
\tilde{C}(h, t)=\frac{9 \mu V^{2}}{128 \pi h^{5}} a(t) .
$$

\section{Solution Procedure}

Given the initial conditions of solder droplet impact, the final solder diameter is obtained by the following procedure, which can be readily implemented using computational software.

a) Based on the initial conditions, compute the time evolution of $h$ for a fully liquid state using (3) until it reaches its minimum. Then $t_{m}$ and $D_{m}$ are obtained straightforwardly.

b) Solve (14) to obtain $t_{1}$.

c) Solve (16) to obtain $t_{2}$.

d) Return to solve (3) until the time reaches $t_{1}$, to determine the height (or the diameter) evolution of a fully liquid droplet.

e) Continue this computation for semi-solid phase using (23) to obtain $h$ (or $D$ ) until the solid fraction reaches 0.625 , when the drop stops moving.

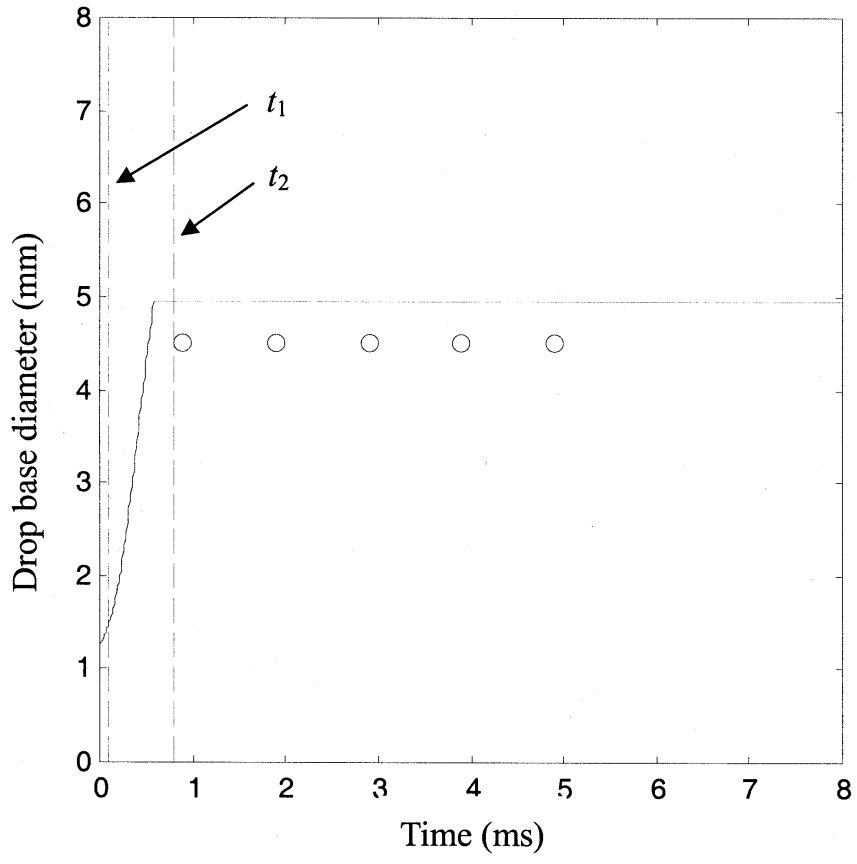

Fig. 2. Modeling and experimental results for a tin droplet on aluminum substrate. In the experiments, original droplet diameter is $1.57 \mathrm{~mm}$, impact velocity $3.04 \mathrm{~m} / \mathrm{s}$, droplet temperature at impact $261{ }^{\circ} \mathrm{C}$, and the substrate temperature $30{ }^{\circ} \mathrm{C}$. The calculation reveals that $t_{1}=0.104 \mathrm{~ms}$ and $t_{2}=0.788 \mathrm{~ms}$.

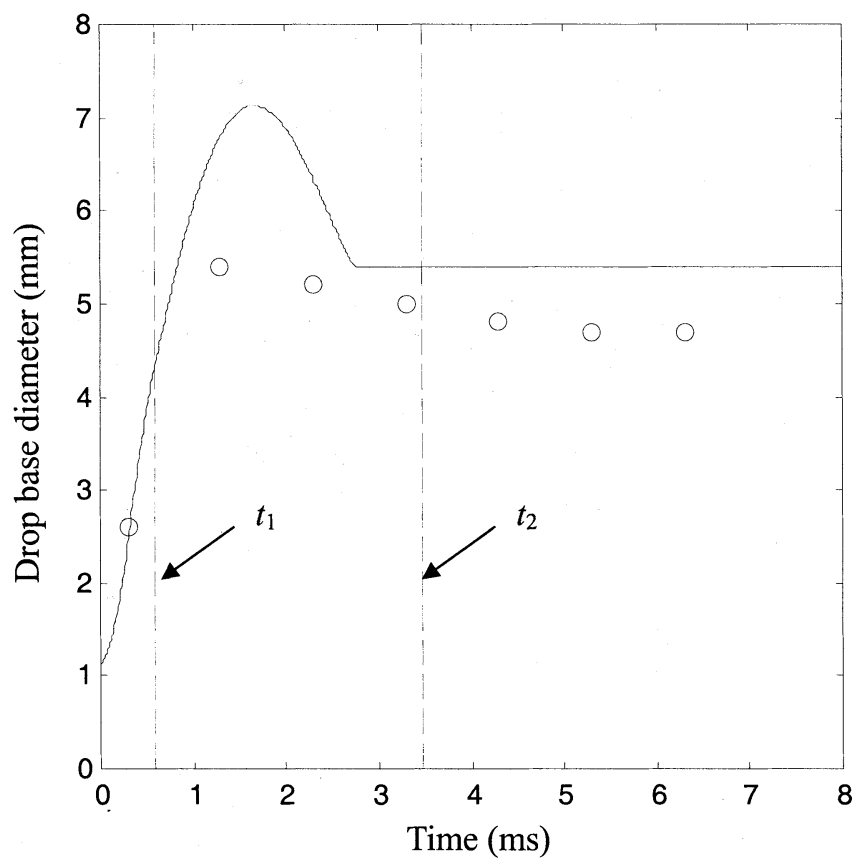

Fig. 3. Modeling and experimental results for a tin droplet on SUS304 substrate. In the experiments, original droplet diameter is $1.40 \mathrm{~mm}$, impact velocity $2.91 \mathrm{~m} / \mathrm{s}$, droplet temperature at impact $257^{\circ} \mathrm{C}$, and the substrate temperature $183{ }^{\circ} \mathrm{C}$. The calculation reveals that $t_{1}=0.593 \mathrm{~ms}$ and $t_{2}=3.473 \mathrm{~ms}$

\section{RESULTS AND DISCUSSION}

To confirm the validity of the model developed above, the simulation results are compared with the experimental data presented in [17], as shown in Figs. 2-4. Pure tin was used as the 


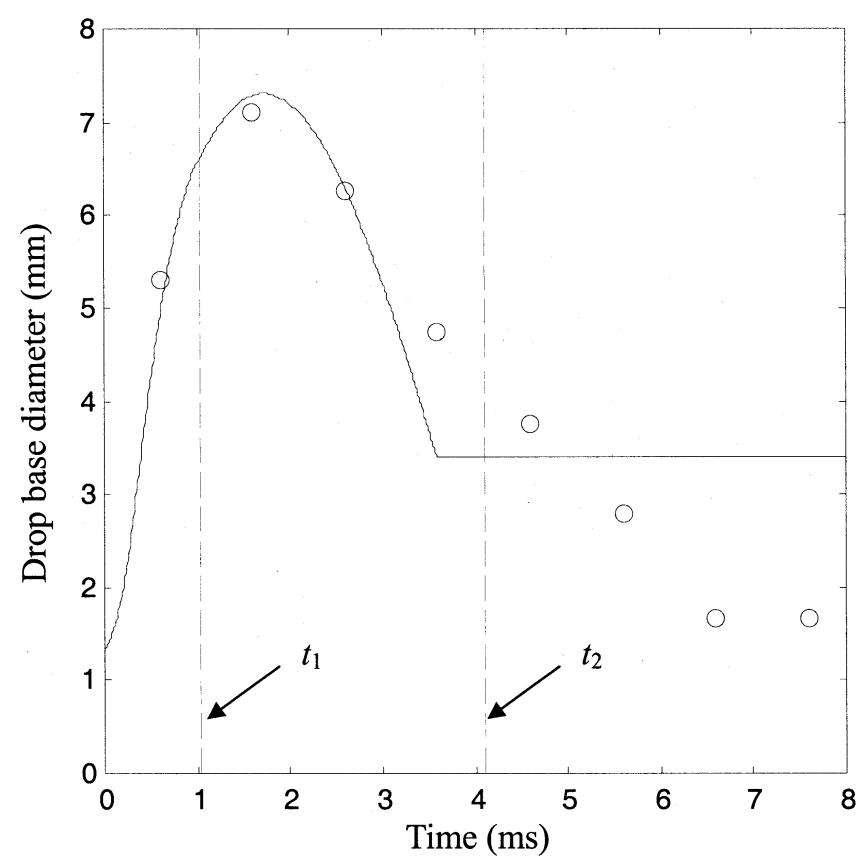

Fig. 4. Modeling and experimental results for a tin droplet on SUS304 substrate. In the experiments, original droplet diameter is $1.67 \mathrm{~mm}$, impact velocity $3.08 \mathrm{~m} / \mathrm{s}$, droplet temperature at impact $263{ }^{\circ} \mathrm{C}$, and the substrate temperature $195{ }^{\circ} \mathrm{C}$. Beyond $t=2.6 \mathrm{~ms}$, the experimental data correspond to the maximum diameter of a droplet, which is in the air due to bouncing, at each moment. The calculation reveals that $t_{1}=1.029 \mathrm{~ms}$ and $t_{2}=4.101 \mathrm{~ms}$.

TABLE I

PHYSICAL PROPERTIES OF LIQUID TIN

\begin{tabular}{lcccc}
\hline $\begin{array}{l}\text { Density } \\
\left(\mathrm{kg} / \mathrm{m}^{3}\right)\end{array}$ & $\begin{array}{c}\text { Viscosity } \\
(\mathrm{Pa} \cdot \mathrm{s})\end{array}$ & $\begin{array}{c}\text { Surface tension } \\
(\mathrm{N} / \mathrm{m})\end{array}$ & $\begin{array}{c}\text { Specific heat } \\
(\mathrm{J} / \mathrm{kg} / \mathrm{K})\end{array}$ & $\begin{array}{c}\text { Heat of fusion } \\
(\mathrm{J} / \mathrm{kg})\end{array}$ \\
\hline 7000 & $1.9 \times 10^{-3}$ & 0.44 & 230 & $5.9 \times 10^{4}$ \\
\hline
\end{tabular}

droplet material in the experiments. Stainless steel 304 and aluminum were the target materials. Images of tin droplets impacting with solid surfaces of various thermal conditions can be found in [17]. Figs. 2 and 3 show that our model succeeds in predicting the final bump diameter within $10 \%$ of discrepancy. Fig. 4 corresponds to a case where the droplet completely bounces off the target surface after impact due to its excessive surface potential energy, but the experimental results prior to the bouncing agree well with the modeling predictions. We note that our model is unable to predict the complete disengagement of the droplet off the substrate. This difficulty is common to other droplet impact simulation programs as well since the total rebounding involves a singular process of the computational domain (droplet) leaving the surface. Table I shows the thermal properties of materials used in the simulation of this work.

Using this model, we performed a series of simulations to evaluate the effects of various process conditions on solder bump shapes. Here we emphasize that it is extremely time consuming and computationally expensive to conduct such evaluations using rigorous numerical procedures which solve the full Navier-Stokes equation and the energy equation. In this work, we evaluate the effects of the temperature of the droplet at impact, the substrate temperature and its thermal properties on the final bump diameter (or height) of pure tin droplets. The
TABLE II

THERMAL PROPERTIES OF THE SUBSTRATES USED IN THE SimULATIONS

\begin{tabular}{ccccc}
\hline Material & $\begin{array}{c}\text { Density } \\
\left(\mathrm{kg} / \mathrm{m}^{3}\right)\end{array}$ & $\begin{array}{c}\text { Heat capacity } \\
(\mathrm{J} / \mathrm{kg} / \mathrm{K})\end{array}$ & $\begin{array}{c}\text { Thermal conductivity } \\
(\mathrm{W} / \mathrm{m} / \mathrm{K})\end{array}$ & $\begin{array}{c}\text { Effusivity } \\
\left(\mathrm{kg} / \mathrm{K} / \mathrm{s}^{5 / 2}\right)\end{array}$ \\
\hline SUS 304 & 7900 & 477 & 15 & 7518 \\
Aluminum & 2702 & 903 & 237 & 24050 \\
Copper & 8933 & 385 & 401 & 37140 \\
Silicon & 2330 & 712 & 148 & 15670 \\
Alumina & 3970 & 765 & 36 & 10460 \\
\hline
\end{tabular}

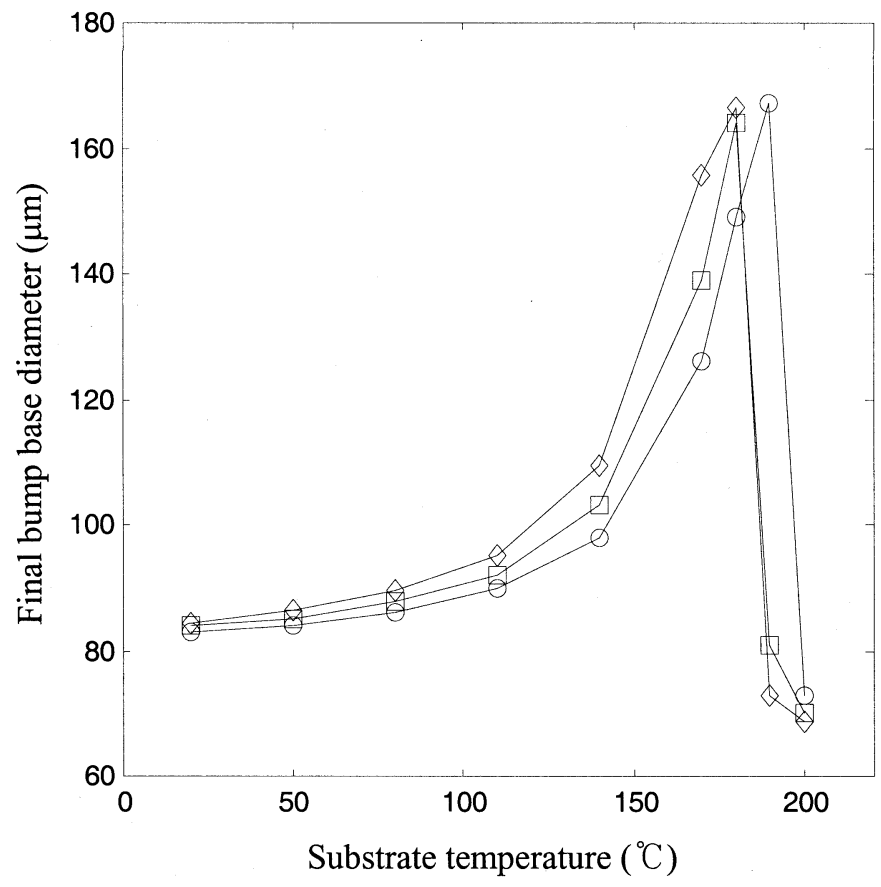

Fig. 5. Final bump base diameter as functions of copper substrate temperature with various initial drop temperature. Circles denote the substrate temperature of $242{ }^{\circ} \mathrm{C}$, squares $262{ }^{\circ} \mathrm{C}$, and diamonds $282{ }^{\circ} \mathrm{C}$.

droplet diameter and impact velocity are $100 \mu \mathrm{m}$ and $1 \mathrm{~m} / \mathrm{s}$, respectively, which are typical in the solder jetting applications. The initial temperature of the droplet varies from $242{ }^{\circ} \mathrm{C}$ to $282{ }^{\circ} \mathrm{C}$ (superheat corresponding to $10{ }^{\circ} \mathrm{C}$ to $50{ }^{\circ} \mathrm{C}$ ). The substrate materials used in the simulation are copper, silicon, and alumina in the descending order of effusivity (Table II). The substrate temperature ranged from room temperature to $200{ }^{\circ} \mathrm{C}$.

In our simulation, a typical impact velocity in the solder jetting process, $1 \mathrm{~m} / \mathrm{s}$, has been chosen thus the corresponding impact Weber number is 1.6. In this low Weber number regime, the effect of the impact velocity variation near the typical process condition upon the spreading dynamics is not significant. The computation results of [4] show that the variation of the impact velocity for microdroplets induces only a subtle change in the final bump shape. Kim and Chun [10] also showed that the recoiling dynamics are entirely governed by the Ohnesorge number rather than the Weber number even when the Weber number is in the order of 100 .

Fig. 5 shows the simulation results of the final bump diameter on a copper substrate while the droplet and substrate temperature vary. Regardless of the droplet temperature, the bump di- 


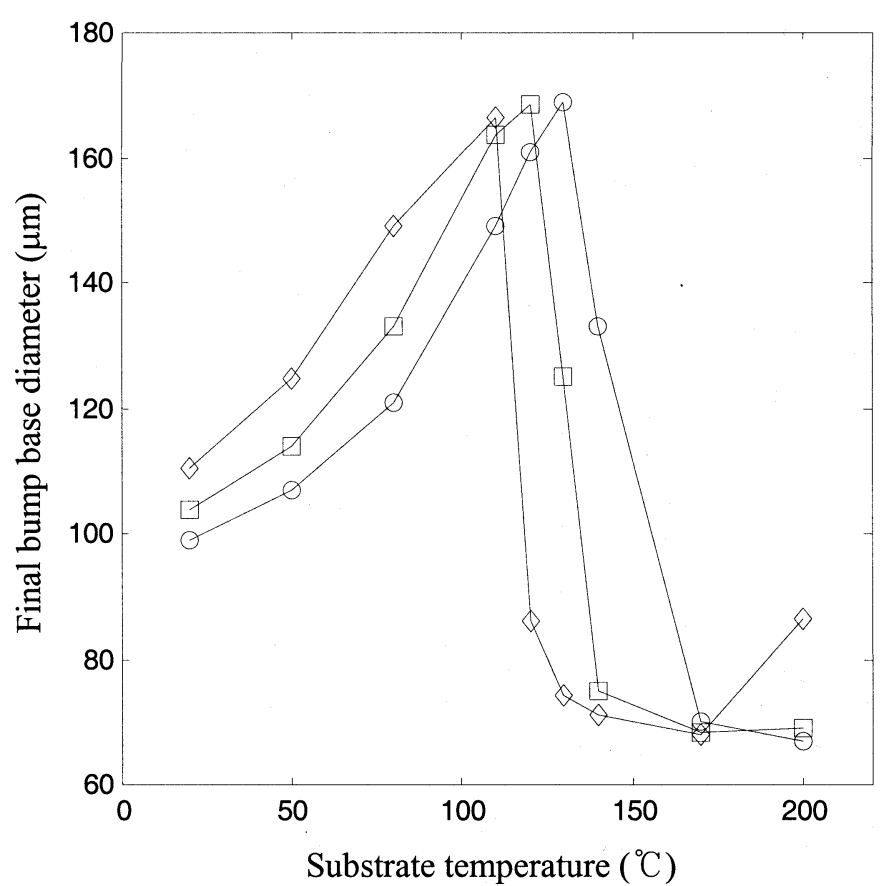

Fig. 6. Final bump base diameter as functions of silicon substrate temperature with various initial drop temperature. Circles denote the substrate temperature of $242{ }^{\circ} \mathrm{C}$, squares $262^{\circ} \mathrm{C}$, and diamonds $282{ }^{\circ} \mathrm{C}$.

ameter increases until the substrate temperature rises to $180^{\circ} \mathrm{C}$. This means that in this substrate temperature range, the droplet stops its motion, i.e., its solid fraction reaches 0.625 , before the recoiling takes place due to a high effusivity of the copper substrate. As the substrate temperature exceeds $180{ }^{\circ} \mathrm{C}$, the bump diameter decreases dramatically due to recoiling of the droplet. It is because the recoiling sets in before freezing arrests the droplet motion. It is also found that the droplet temperature has negligible effects on the final bump diameter. This implies that the droplet rapidly cools to its freezing temperature upon impact due to a high effusivity of copper, thus the superheat plays a minor role in the droplet's thermal state change. When the copper substrate temperature is quite high, it may react with the tin drop, rendering the problem beyond the scope of the current analysis. Further study is called for to describe the impact of a drop with a chemically reactive substrate.

The simulated bump diameter on a silicon substrate with varied droplet and substrate temperatures is shown in Fig. 6. The bump diameter increases with the rise of the substrate temperature up to about $120^{\circ} \mathrm{C}$, and the bump diameter rapidly decreases in higher temperature ranges. We note that the substrate temperature at which the bump diameter reaches the maximum for silicon substrate $\left(120^{\circ} \mathrm{C}\right)$ is lower than that for copper substrate $\left(180^{\circ} \mathrm{C}\right)$. This is because the freezing is slow enough to let the recoiling start before the droplet ceases its motion when the substrate temperature is higher than $120{ }^{\circ} \mathrm{C}$ instead of $180^{\circ} \mathrm{C}$, due to a lower effusivity of silicon. Another point which distinguishes Fig. 6 from Fig. 5 is that the droplet impact temperature has a significant influence on the final bump diameter. This suggests that the droplet superheat plays an important role because the droplet does not reach its freezing point as rapidly as it does on a copper substrate. In addition, the substrate temperature, above which the recoiling takes effect

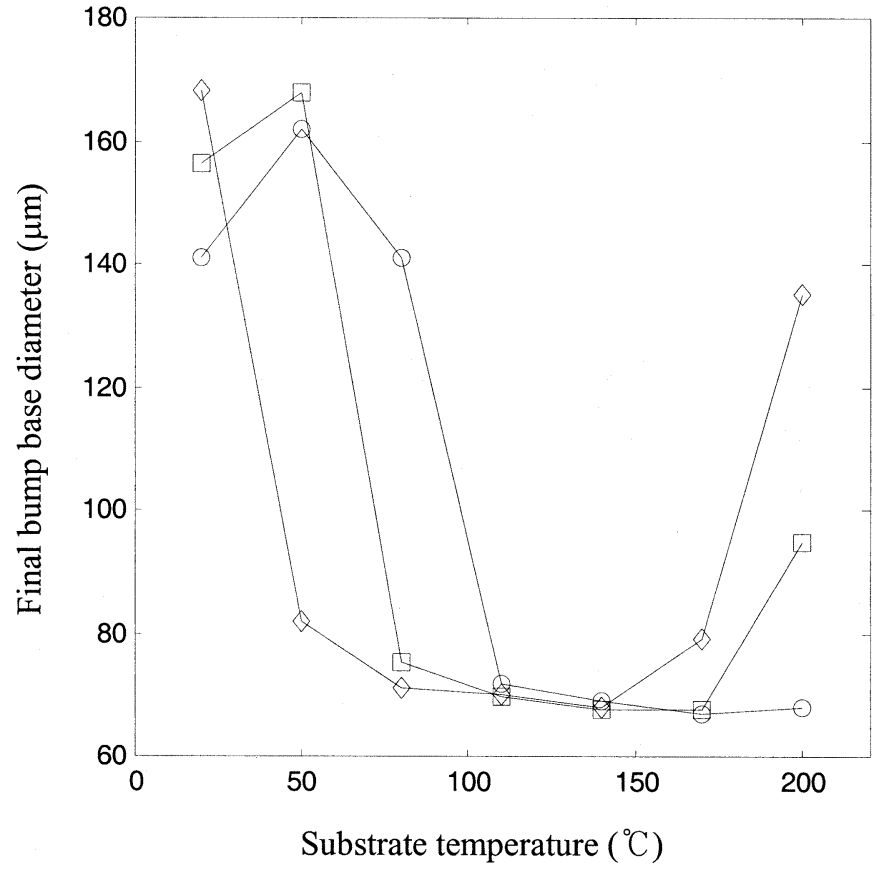

Fig. 7. Final bump base diameter as functions of alumina substrate temperature with various initial drop temperature. Circles denote the substrate temperature of $242{ }^{\circ} \mathrm{C}$, squares $262^{\circ} \mathrm{C}$, and diamonds $282^{\circ} \mathrm{C}$.

before droplet is arrested, decreases as the droplet temperature increases. It is also interesting to note that when the droplet temperature is $282{ }^{\circ} \mathrm{C}$, the bump diameter increases again at the substrate temperature of $200{ }^{\circ} \mathrm{C}$. It is because the droplet re-spreads after recoiling owing to its long-lasting motility for slow freezing.

Fig. 7 shows the simulated bump diameter on alumina $\left(\mathrm{Al}_{2} \mathrm{O}_{3}\right)$ substrate which possesses an even lower effusivity than silicon. The substrate temperature that results in the maximum bump diameter is much lower than that of the aforementioned substrates because of the low effusivity of alumina. Especially for the droplet temperature of $282{ }^{\circ} \mathrm{C}$, even a slight heating of the substrate induces recoiling before arrest, which greatly reduces the final bump diameter. In this case, the effects of the droplet temperature are more profound as can be seen through greatly different shapes of curves for varying droplet temperature. Bumps re-spread after recoil when the substrate temperature approaches $200^{\circ} \mathrm{C}$ for droplet temperatures higher than $262{ }^{\circ} \mathrm{C}$.

\section{CONCLUSION}

In this work, we develop a model that simulates the spreading and solidification of molten solder droplets on subcooled target surfaces. Our model uses the variational principle to solve the fluid flow. The heat transfer problem is decoupled from that of fluid flow. The motion of a semi-solid phase is analyzed assuming a non-Newtonian slurry flow. A significant computational effort is saved by solving two second-order differential equations successively instead of solving the full Navier-Stokes equation with the moving boundary and the energy equation. This simple modeling enables the quick evaluation of the effects of various process parameters upon final bump diameter. 
Here we investigated the effects of such parameters as droplet impact temperature, substrate materials, and substrate temperature on the final diameter of pure tin bumps.

Summarizing the simulation results above, the droplets deposited on high-effusivity substrates freeze rapidly so that the effects of recoiling do not emerge until the substrate temperature is significantly raised. In this case, the droplet superheat has only minor effects on the final bump diameter. However, as the effusivity of the substrate decreases, the freezing slows and the recoiling tends to affect the final bump diameter in a wide range of substrate temperature. Moreover, the droplet superheat becomes greatly important since the droplet does not reach its freezing point as rapidly as ones on high-effusivity substrates.

Based on this model, it is possible to predict and further, control the bump diameter deposited on various substrates with great ease as compared to conventional numerical procedures. Our major contribution lies in providing an analysis tool that can be used together with the experimental efforts to find the optimum process conditions that yield the desired bump geometry. The simulation results presented in this work suggest that when the substrate possesses a high effusivity, the substrate temperature is a single dominant controlling parameter that determines the final bump diameter. However, when the effusivity of a substrate is relatively low, both the substrate temperature and the drop superheat should be tailored together to obtain the desired bump geometry. Moreover, merely increasing the substrate temperature does not guarantee the monotonic decrease or increase of the bump diameter because recoiling and re-spreading may take place depending on the solidification rate.

\section{REFERENCES}

[1] N.-C. Lee, "The use of solder as an area-array package interconnect," Chip Scale Rev., Sept./Oct. 1999.

[2] J.-H. Chun and C. H. Passow, "Droplet-Based manufacturing," CIRP Annals, vol. 42/1, pp. 235-238, 1993.

[3] J. M. Waldvogel, D. Poulikakos, D. B. Wallace, and R. Marusak, "Transport phenomena in picoliter size solder droplet dispension," J. Heat Transfer, vol. 118, pp. 148-156, 1996.

[4] J. M. Waldvogel and D. Poulikakos, "Solidification phenomena in picoliter size solder droplet deposition on a composite substrate," Int. J. Heat Mass Transfer, vol. 40, pp. 295-309, 1997.

[5] B. Xiong, C. M. Megaridis, D. Poulikakos, and H. Hoang, "An investigation of key factors affecting solder microdroplet deposition," J. Heat Transfer, vol. 120, pp. 259-270, 1998.

[6] M. Pasandideh-Fard, R. Bhola, S. Chandra, and J. Mostaghimi, "Deposition of tin droplets on a steel plate: Simulations and experiments," Int. J. Heat Mass Transfer, vol. 41, pp. 2929-2945, 1998.

[7] J. Madejski, "Solidification of droplets on a cold surface," Int. J. Heat Mass Transfer, vol. 19, pp. 1009-1013, 1976.

[8] T. Watanabe, I. Kuribayashi, T. Honda, and A. Kanzawa, "Deformation and solidification of a droplet on a cold substrate," Chem. Eng. Sci., vol. 47, pp. 3059-3065, 1992.
[9] H. Fukanuma, "A porosity formation and flattening model of an impacting molten particle in thermal spray coatings," J. Thermal Spray Tech., vol. 3, pp. 33-44, 1994.

[10] H.-Y. Kim and J.-H. Chun, "The recoiling of liquid droplets upon collision with solid surfaces," Phys. Fluids, vol. 13, pp. 643-659, 2001.

[11] P. A. Joly, "Rheological Properties and Structure of a Semi-Solid Tin-Lead Alloy,” Ph.D., Dept. Metallurgy Mater. Sci., MIT, Cambridge, MA, 1974.

[12] S. H. Crandall, D. C. Karnopp, E. F. Kurtz Jr., and D. C. Pridmore-Brown, Dynamics of Mechanical and Electromechanical Systems. New York: McGraw-Hill, 1968.

[13] H. S. Carslaw and J. C. Jaeger, Conduction of Heat in Solids, 2nd ed. Oxford, UK: Oxford Univ. Press, 1959.

[14] P. A. Joly and R. Mehrabian, "The rheology of a partially solid alloy," J. Mater. Sci., vol. 11, pp. 1393-1418, 1976.

[15] P. Kumar, C. L. Martin, and S. Brown, "Flow behavior of semi-solid alloy slurries," in Proc. 2nd Int. Conf. Semi-Solid Process Alloys Composites, 1992, pp. 248-262.

[16] N. A. Frankel and A. Acrivos, "On the viscosity of concentrated suspension of solid spheres," Chem. Eng. Sci., vol. 22, pp. 847-853, 1967.

[17] H.-Y. Kim, "Spreading Behavior of Molten Metal Microdroplets," Ph.D., Dept. Mech. Eng., MIT, Cambridge, MA, 1999.

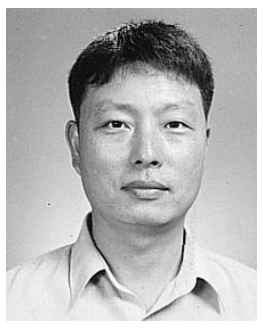

Young-Soo Yang received the M.S. and Ph.D. degrees from the Korea Advanced Institute of Science and Technology, Seoul, in 1987 and 1991, respectively, both in production engineering.

At present, he is an Associate Professor of mechanical engineering in Chonnam National University, Gwangju, Korea.

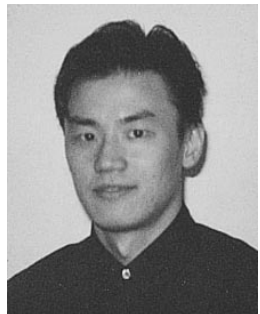

Ho-Young Kim received the B.S. degree from Seoul National University, Seoul, Korea, in 1994 and the S.M. and Ph.D. degrees from the Massachusetts Institute of Technology, Cambridge, in 1996 and 1999, respectively, all in mechanical engineering.

$\mathrm{He}$ is currently at the Thermal/Flow Control Research Center, Korea Institute of Science and Technology, Seoul. His research interest centers around microfluidics and interfacial flow physics.

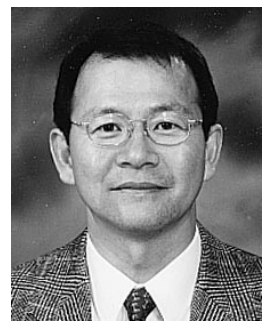

Jung-Hoon Chun received the B.S. degree from Seoul National University, Seoul, Korea, the M.A.Sc. degree from the University of Ottawa, Ottawa, ON, Canada, and the Ph.D. degree from the Massachusetts Institute of Technology (MIT), Cambridge, all in mechanical engineering.

At present, he is a Professor of mechanical engineering at MIT and Co-Director of the Manufacturing Institute. 